\title{
Effect of frequency detuning on pulse propagation in one-dimensional photonic crystal with a dense resonant medium: application to optical logic
}

\author{
Denis V. Novitsky ${ }^{*}$ \\ B.I. Stepanov Institute of Physics, National Academy of Sciences of Belarus, \\ Nezavisimosti Avenue 68, 220072 Minsk, Belarus.
}

\begin{abstract}
We consider propagation of light pulses detuned from the atomic resonance in a dense twolevel medium and photonic structures with it. The large density of the medium is important to decrease spatial scale of such nonlinear effects as pulse compression, though it does not provide any fundamentally new phenomena as compared to dilute media. Frequency detuning decreases the effectivity of such nonlinear phenomena as pulse compression and dispersion spreading compensation as well. We propose simple logic gates based on interaction between two pulses in one-dimensional nonlinear photonic crystal. It turned out, that frequency detuning is necessary to obtain ultrafast AND gate, while OR and NOT gates can be realized in the system without detuning.

PACS numbers:
\end{abstract}

\section{INTRODUCTION}

A dense resonant medium is a model of two-level medium which takes into account near dipole-dipole (NDD) interactions between atoms. NDD interactions described by local field correction lead to appearance of intrinsic optical bistability 1, 2, 3] or change the characteristics of such effects as optical switching [4, 5], soliton formation [6, 7], etc.

NDD interactions effects are often treated as a result of nonlinear dynamic shift of atomic resonance from the frequency of input radiation. The efficiency of this process is governed by the value $b=4 \pi \mu^{2} C / 3 \hbar \gamma_{2}$, where $\mu$ is the transition dipole moment, $\hbar$ is the Planck constant, $\gamma_{2}=1 / T_{2}$ is the rate of transverse relaxation. The quantity $b$ is usually referred to as a constant of NDD interactions and is limited by the volume density of two-level atoms $C$. Though the magnitude of $b$ is extremely important, the static detuning $\Delta \omega$ of light frequency from atomic resonance should be taken into account as well, as it determines existence regions for a number of phenomena in dense resonant media. For example, local intrinsic optical bistability (IOB) occurs in thin films if the parameter of NDD interactions satisfies the condition [8]

$$
b>2 \delta^{\prime}+6\left(1+\delta^{\prime 2}\right)^{1 / 3} \operatorname{Re}\left(\delta^{\prime}+i\right)^{1 / 3},
$$

so that its minimal value required for IOB amounts to 4 [9] at the detuning $\delta^{\prime}=\Delta \omega / \gamma_{2}=-1$. Another effect is connected with appearance of the so-called incoherent solitons which require static detuning to be negative [7]. On the other hand, detuning should not be too large: if it is much greater than NDD interaction parameter, the resonant medium behaves as a dilute one [10]. This is the reason why it is often assumed to be zero in the problem of ultrashort pulse propagation through the dense resonant medium.

\footnotetext{
*Electronic address: dvnovitsky@tut.by
}

Since in existing literature there is no consistent analysis of static frequency detuning influence on pulse propagation in dense resonant medium, we try to fill up this gap in the present paper. We consider coherent pulse transformation in a dense resonant medium with realistic values of NDD interaction parameter and apply nonzero detunings which are large enough to cause significant effects. Another aspect of the present research, which is of particular interest, is connected with pulse propagation in nonuniform dense medium. Periodic variations of its linear parameters form the one-dimensional photonic crystal. The problem of enhancement of nonlinear pulse transformation effectiveness and pulse controlling by another pulse in such systems is also investigated for the case of nonzero detuning.

The paper contains three sections. In Sec. II the main equations and parameters are considered, as well as the problem of medium density. The peculiarities of pulse compression in a dense resonant medium and photonic crystal with it in the case, when frequency detuning is present, are discussed in Sec. III] Sec. [V] is devoted to a perspective from practical viewpoint result connected with interaction between two pulses - realization of optical logic elements on the basis of nonlinear photonic crystal. As it is demonstrated, detuning of nonlinear medium from resonance plays crucial role allowing to make the operation of logic gates faster and more stable.

\section{DENSE RESONANT MEDIUM: IMPORTANCE OF DENSITY}

Dynamics of pulse propagation in dense resonant medium are described in semiclassical approach by the 
system of generalized Maxwell-Bloch equations [10, 11]:

$$
\begin{aligned}
\frac{d P}{d t} & =\frac{i \mu}{\hbar} E N+i P\left(\Delta \omega+\frac{4 \pi \mu^{2} C}{3 \hbar} N\right)-\gamma_{2} P, \\
\frac{d N}{d t} & =2 \frac{i \mu}{\hbar}\left(E^{*} P-P^{*} E\right)-\gamma_{1}(N-1), \\
\frac{\partial^{2} \Sigma}{\partial z^{2}} & -\frac{1}{c^{2}} \frac{\partial^{2} \varepsilon_{b g} \Sigma}{\partial t^{2}}=\frac{4 \pi}{c^{2}} \frac{\partial^{2} P_{n l}}{\partial t^{2}}
\end{aligned}
$$

where $N$ is the population difference, $P$ is the microscopic (atomic) polarization; $\gamma_{1}=1 / T_{1}$ is the rate of longitudinal relaxation; $c$ is the light speed in vacuum; $k=\omega / c$ is the wavenumber, and $\varepsilon_{b g}$ is the background dielectric permittivity (linear and dispersionless). Macroscopic electric field $\Sigma$ is expressed via its amplitude $E$ as $\Sigma=E \exp [-i(\omega t-k z)]$; similarly for macroscopic nonlinear polarization we have $P_{n l}=\mu C P \exp [-i(\omega t-k z)]$.

The system of equations (2-4) can be written in dimensionless form as follows:

$$
\begin{aligned}
& \frac{d P}{d \tau}= i \tilde{\Omega} N+i P(\tilde{\delta}+\epsilon N)-\tilde{\gamma}_{2} P \\
& \frac{d N}{d \tau}= 2 i\left(\tilde{\Omega}^{*} P-P^{*} \tilde{\Omega}\right)-\tilde{\gamma}_{1}(N-1) \\
& \frac{\partial^{2} \tilde{\Omega}}{\partial \xi^{2}}- \varepsilon_{b g} \frac{\partial^{2} \tilde{\Omega}}{\partial \tau^{2}}+2 i \frac{\partial \tilde{\Omega}}{\partial \xi}+2 i \varepsilon_{b g} \frac{\partial \tilde{\Omega}}{\partial \tau}+\left(\varepsilon_{b g}-1\right) \tilde{\Omega} \\
&=3 \epsilon\left(\frac{\partial^{2} P}{\partial \tau^{2}}-2 i \frac{\partial P}{\partial \tau}-P\right)
\end{aligned}
$$

$\tau=\omega t$ and $\xi=k z$ are dimensionless time and space arguments; $\tilde{\Omega}=(\mu / \hbar \omega) E$ is the dimensionless amplitude of electric field; $\tilde{\delta}=\Delta \omega / \omega$ is the normalized static frequency detuning; $\epsilon=b \tilde{\gamma}_{2} ; \tilde{\gamma}_{j}=\gamma_{j} / \omega, j=1,2$; and $\varepsilon_{b g}$ is the background dielectric permittivity.

In the present paper the input signals are assumed to be coherent Gaussian pulses with amplitude $\tilde{\Omega}=$ $\tilde{\Omega}_{0} \exp \left(-t^{2} / 2 t_{p}^{2}\right)$, i.e., for pulse duration $t_{p}$, the inequality is valid: $t_{p}<<T_{2}<T_{1}$. In our calculations the parameters of the medium and pulse are as follows: $T_{1}=1000 \mathrm{ps}$ and $T_{2}=100 \mathrm{ps}$, pulse duration $t_{p}=30 \mathrm{fs}$ and the wavelength $\lambda=0.5 \mu \mathrm{m}$. Pulse amplitude can be measured in the units of $\Delta \Omega_{T}=\lambda / 2 \sqrt{2 \pi} c t_{p}$, which is a natural parameter connected with optical switching [12]. A pulse with $\tilde{\Omega}_{0}=\Delta \Omega_{T}$ inverts the medium and then returns it exactly to the ground state, so that it can be treated as a $2 \pi$-pulse. Periodic variation of background dielectric constant $\varepsilon_{b g}$ describes the situation of one-dimensional nonlinear photonic crystal. In order to accurately take into account processes connected with dispersion, the numerical simulation of the system of equations (5.7) is implemented on the basis of approach developed in 10, 12].

The additional nonlinear term with $\epsilon N$ in Eq. (5), which couples polarization and population difference, is responsible for near dipole-dipole interactions. As it was shown in Ref. [12], influence of this term on coherent pulse propagation (in contrast to consideration in stationary regime) is negligible at realistic values of the parameter $b$ which is usually limited to several units (at best, $b=10$ ). Though it will be the case further, the dense resonant medium has a very important advantage over dilute media. The density of the medium is present in the right hand side of the wave equation (7) due to the macroscopic polarization definition (it measures the dipole moment of macroscopic volume) and, hence, influences on pulse propagation. This influence is not physically connected with NDD interactions (formally, there is just the same combination of parameters $\epsilon$ ) and determines spatial scale of nonlinear effects in the resonant two-level medium. High values of the density and, consequently, of the constant $b$ allow to obtain such phenomena as pulse compression on significantly reduced distances. Indeed, the distance of optimal compression (limited by the processes of diffraction, dispersion and energy absorbtion) [12] approximately equals $1000 \lambda$ for $b=1$, while for $b=10$ it is only about $100 \lambda$ (for the pulse with $\tilde{\Omega}=1.5 \Delta \Omega_{T}$ ). Thus, the large density of resonant medium is important to increase effectiveness of nonlinear effects and compactness of their applications due to right hand side of Eq. (7), though it does not provide any fundamentally new phenomena connected with NDD interactions term in Eq. (5).

The previous results [12] were obtained for the case of exact resonance, i.e. detuning $\tilde{\delta}$ of the field frequency from atomic resonance was zero. Further we consider influence of nonzero detuning on pulse compression in the dense resonant medium and photonic crystal containing it.

\section{EFFECT OF FREQUENCY DETUNING ON PULSE COMPRESSION}

Let us consider propagation of short coherent $\left(t_{p}<<\right.$ $T_{2}$ ) pulse in a dense resonant medium with NDD interaction constant $b=10$ and background permittivity $\varepsilon_{b g}=1$ (other parameters are stated in the previous section), and pulse intensity is high enough to obtain inversion of medium, i.e. $\tilde{\Omega}_{0} \sim \Delta \Omega_{T}$. It is easy to assume that influence of frequency detuning $\tilde{\delta}$ becomes significant when it is comparable with this dimensionless value $\Delta \Omega_{T}$, too. This is proved by Fig. 1 (here and further pulse amplitude and detuning of the medium are measured in the units of $\Delta \Omega_{T}$, i.e. $\delta=\tilde{\delta} / \Delta \Omega_{T}$ and $\left.\Omega=\tilde{\Omega} / \Delta \Omega_{T}\right)$. It is seen that, when increasing detuning from the resonance, the final quasistationary level of population difference is shifted from the fully inverted state (if detuning is absent) almost to the ground state (for $\delta=2$ ). At the same time the effectivity of pulse compression is decreasing (see Fig. 1b). The sign of frequency detuning almost does not affect the behavior of pulse in the dense resonant medium, according to the comparison of results for $\delta=1$ and $\delta=-1$ demonstrated in Fig. 2, Note that the detuning $|\delta|=1$ corresponds (for parameters used) to the frequency shift of about $\Delta \omega \approx 0.011 \omega$.

Nevertheless, the same or even higher pulse compression can be reached for more thick layers. This means 

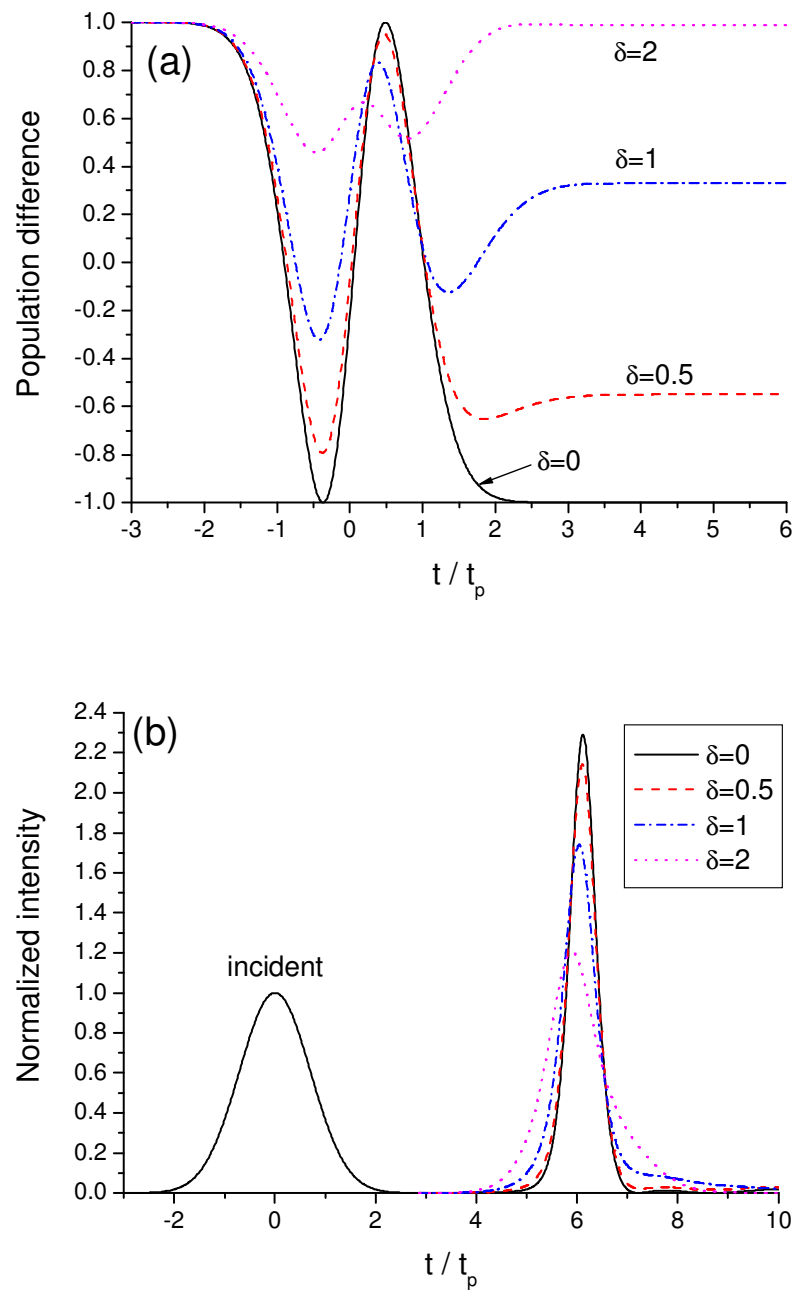

FIG. 1: (Color online) (a) Dynamics of population difference on the entrance of the layer of the dense resonant medium after pulse passage and (b) transmitted pulse form transformation for different values of frequency detuning $\delta$. The amplitude of pulse is $\Omega_{0}=1.5$. Layer thickness is $L=100 \lambda$.

that, for $\delta \sim 1$, longer distances are needed to provide effective nonlinear interaction of pulse with the dense resonant medium and, hence, the distance of optimal compression (limited by diffraction and dispersion) is increasing, as well. For example, it amounts to about $100 \lambda$ for $\delta=0$, while for $\delta=1$ it is approximately $250 \lambda$ (see Fig. 3).

Another negative influence of frequency detunings is connected with the effect of compensation of dispersion spreading in nonlinear photonic crystals found in Ref. [12]. While pulse spreads rapidly as it propagates in linear photonic crystal (which is an element with high level of dispersion), nonlinear compression allows to compensate this widening. Fig. 4 shows that this property is significantly reduced for $\delta=1$ (several low-intensive pulses appear instead of a single high-intensive one) and, especially, for $\delta=-1$ (one broadened low-intensive pulse).

Thus, off-resonant interaction of a single light pulse

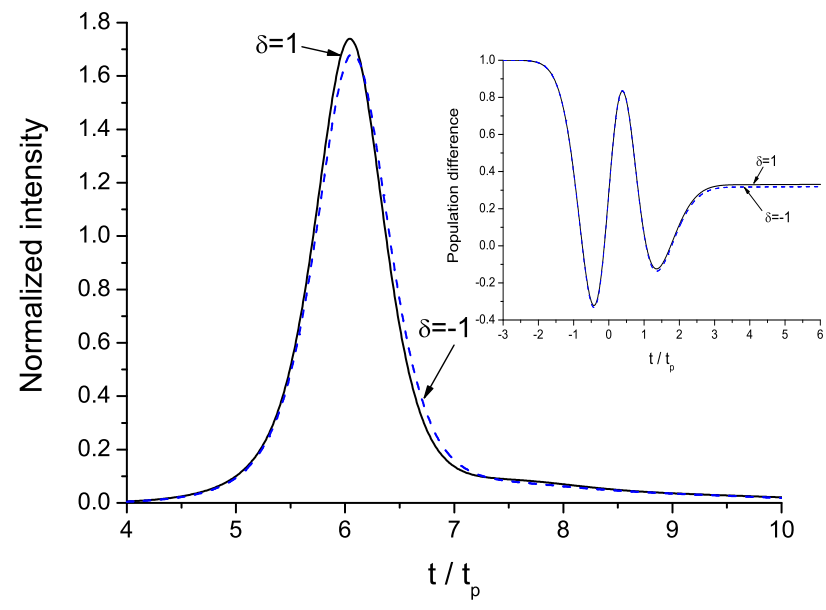

FIG. 2: (Color online) Comparison of transmitted pulse forms for differnt signs of frequency detuning $\delta$. The amplitude of pulse is $\Omega_{0}=1.5$. Layer thickness is $L=100 \lambda$. The inset shows corresponding dynamics of population difference.

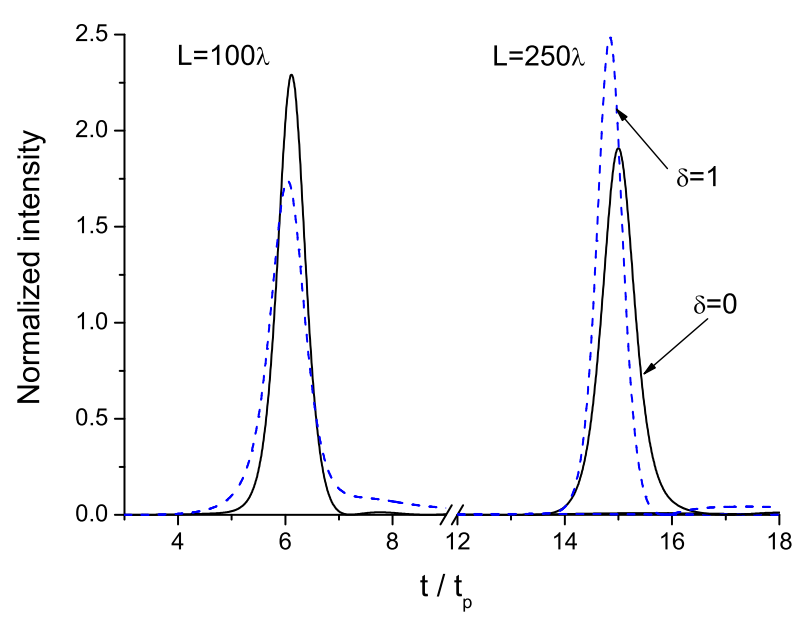

FIG. 3: (Color online) Comparison of transmitted pulse forms for different frequency detunings $\delta$ at different layer thicknesses. The amplitude of pulse is $\Omega_{0}=1.5$.

with high enough detunings $\delta \sim 1$ with the dense twolevel medium results in decrease of efficiency of such important processes as pulse compression and dispersion spreading compensation. However, frequency detuning is turned out to be useful or even necessary condition for two-pulse interactions and, in particular, for realization of optical logic elements on the basis of nonlinear photonic structure.

\section{OPTICAL LOGIC GATES}

In Ref. 12] it was shown that pulse intensity can be controlled with another pulse in the scheme of two pulses copropagating in a resonant two-level medium. Moreover, using of photonic crystal allows to significantly enhance the efficiency of this process. This phenomenon is 

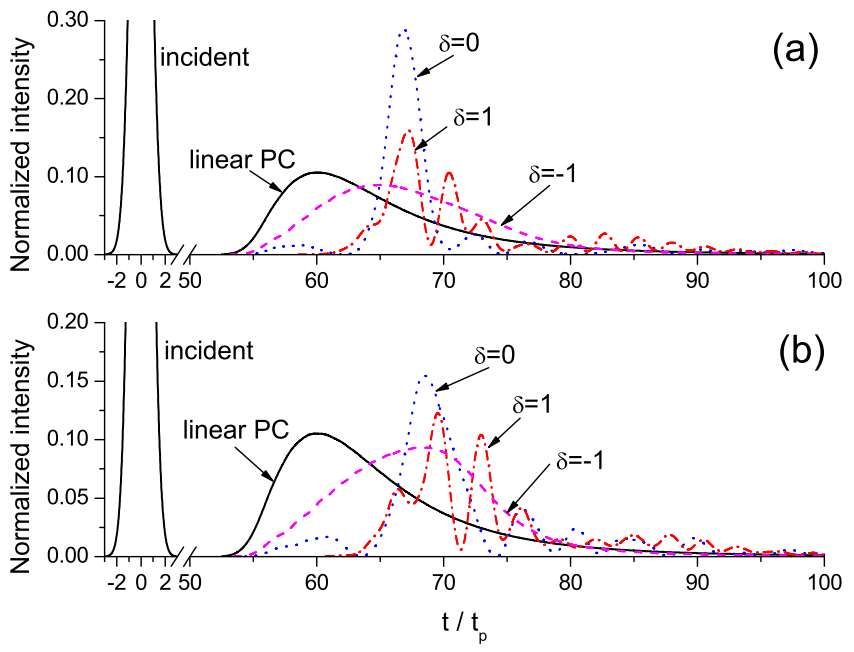

FIG. 4: (Color online) The forms of pulses transmitted through the photonic crystal with (a) nonlinear layers $d_{1}$, (b) both nonlinear layers $d_{1}$ and $d_{2}$ at different values of frequency detuning $\delta$. The number of periods is 250, the thicknesses $d_{1}=0.4, d_{2}=0.13 \mu \mathrm{m}$, background refractive indices $n_{1}=1$, $n_{2}=3.5$. The amplitude of pulse is $\Omega_{0}=1.5$.

a basic idea for optical logic elements to be considered.

Possibility of optical logic operations is one of the most prospective results in nonlinear optics of photonic crystals. Usually logic gates are realized by using two- and three-dimensional photonic band-gap structures with defects which form waveguides and nonlinear resonators (see, e.g., [13, 14] and references there). Such systems must have, at least, two inputs for initial signals and one output for resulting one. It seems to be much more simple to use one-dimensional structures with the same entry for both input signals. An example of one-dimensional logic gates on the basis of nonlinear semiconductor heterostructure was given in [15]. The possibility of onedimensional logic elements based on photonic crystal with dense resonant medium is to be discussed further.

The important property of the gates proposed is that the same nonlinear structure can serve for different logic gates, depending on the intensity of input signals. (Similar property was used in [15] to demonstrate optical elements in stationary regime.) The first pulse changes the characteristics of nonlinear medium, as it propagates in it, while the second one interacts with this modified medium. This effect can be made more sharp by using photonic crystal. In this case specific reflection and transmission properties of this structure become essential.

First, let us consider the case $\delta=0$. Fig. 5 shows the example of numerical simulation of Eqs. (5,7) for the photonic crystal with reflectivity $R=0.469$ at the main wavelength $\lambda$. Different logic operations can be achieved for different levels of input intensities. OR gate (disjunction) corresponds to the case when, in both cases of one and two pulses on the entrance, one obtains approximately the same peak intensity on the output. This situation occurs for the input pulses with the intensities
TABLE I: Table of output peak intensities (truth tables)

\begin{tabular}{l|ccc|cc}
\hline Gate at $\delta=0$ & $\Omega_{1}$ & $\Omega_{2}$ & $\delta=0$ & $\delta=1$ & $\delta=-1$ \\
\hline 1. OR & 0 & 0 & 0 & 0 & 0 \\
& 1 & 0 & 0.28 & 0.14 & 0.07 \\
& 0 & 1 & 0.28 & 0.14 & 0.07 \\
& 1 & 1 & 0.26 & 0.19 & 0.37 \\
\hline 2. AND & 1.5 & 0 & 0.37 & 0.27 & 0.23 \\
& 0 & 1.5 & 0.37 & 0.27 & 0.23 \\
& 1.5 & 1.5 & 0.90 & 0.84 & 1.01 \\
\hline 3. NOT & 0 & 1 & 0.28 & 0.14 & 0.07 \\
& 0.5 & 1 & 0.09 & 0.11 & 0.19 \\
\hline 4. OR & 1.5 & 0 & 0.37 & 0.27 & 0.23 \\
& 0 & 1 & 0.28 & 0.14 & 0.07 \\
& 1.5 & 1 & 0.33 & 0.60 & 0.70 \\
\hline 5. AND & 1 & 0 & 0.28 & 0.14 & 0.07 \\
& 0 & 1.5 & 0.37 & 0.27 & 0.23 \\
& 1 & 1.5 & 0.85 & 0.70 & 0.70 \\
\hline
\end{tabular}

$\Omega_{1}=\Omega_{2}=1$ (in units of $\Delta \Omega_{T}$ ) and is demonstrated in Fig. 5 k. The results for transmission of these pulses are summed up in the section 1 of Table I. It is possible to say that this section represents a truth table for the OR element considered.

AND gate (conjunction) occurs when, as a result of nonlinear interaction of two pulses, the intensity of the transmitted one is much greater than in the case of a single pulse. This situation takes place for $\Omega_{1}=\Omega_{2}=1.5$ and is shown in Fig. 5b. The corresponding truth table (Table I. section 2) shows that the state when both signals are present (upper or AND-state) gives almost 2.5times higher peak output intensity than the state when one of them is absent (lower state).

Finally, NOT operation can be realized as follows. Propagation of a single pulse $\Omega_{2}=1$ gives the output peak intensity of about 0.28 (see Fig. 5 r), so that it can be considered as ON-state. By using another pulse of $\Omega_{1}=0.5$, the output intensity is dramatically decreased to 0.09 , i.e. more than by a factor of three. This low level of transmitted intensity corresponds to the OFFstate. This effect of switching between ON- and OFFstates forms the basis of NOT gate. Its truth table is shown in section 3 of Table [

As it is seen from the Table [1 the gates also can be realized for other combinations of input pulse intensities. For example, OR gate occurs when $\Omega_{1}=1.5, \Omega_{2}=1$, while for the reverse case $\left(\Omega_{1}=1, \Omega_{2}=1.5\right)$ one obtains AND operation.

As it was mentioned, result of interaction between pulses strongly depends on the properties of photonic band-gap structure and, in particular, on its reflectivity. For example, if we take photonic crystal with large linear reflectivity, namely $R=0.825$ (it can be made by changing the thickness $d_{2}$ to $0.1274 \mu \mathrm{m}$ ), logic operations described above become impossible. For the pulses with the amplitude $\Omega_{1}=\Omega_{2}=1.5$ (it was the AND gate in Fig. 5b) the results of pulse propagation are as 

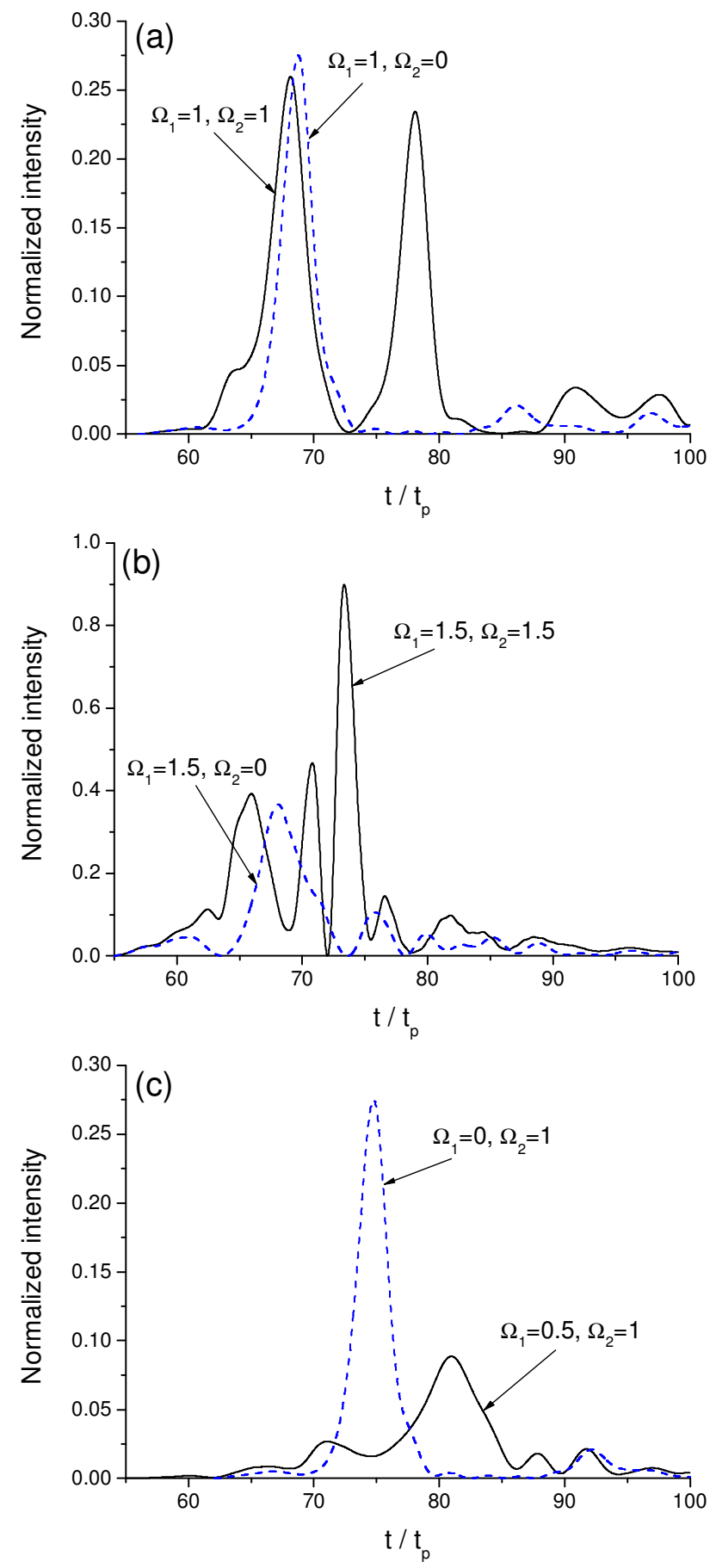

FIG. 5: (Color online) Shapes of transmitted pulses for different input signals corresponding to (a) OR gate, (b) AND gate, (c) NOT gate. The number of periods is 250, the thicknesses of the layers $d_{1}=0.4 \mu \mathrm{m}, d_{2}=0.1301 \mu \mathrm{m}$, background refractive indices $n_{1}=1, n_{2}=3.5$. Frequency detuning is $\delta=0$.

follows: output intensity of 0.42 for a single pulse and 0.53 for two pulses, i.e. there is no pronounced ANDstate. The contrast between states in other cases gets less sharp, too. Thus, realization of the optical logic op- erations is the result of consistent change of pulse intensities and nonlinear photonic crystal properties: during propagation, pulse changes the characteristics of nonlinear photonic band-gap structure, which, in turn, has an influence on the pulse intensities. This mutual change leads to complexity of the effect of logic operations and nonobviousness of its appearance at certain parameters of the system.

Now let us consider the results of simulations for the case $|\delta|=1$ which are represented in Table I (two last columns). It is seen that frequency detuning increases the contrast between lower and upper states of the AND gate. Moreover, OR and NOT gates (at $\delta=0$ ) become less pronounced or even transform to the AND gate when the detuning is present. This is especially typical for negative detuning $\delta=-1$. Thus, the system with detuning can be used to obtain high-contrast AND operation, while OR and NOT gates are realized at $\delta=0$. It is important, that AND gate with detuning has another advantage connected with its bit rate.

Since the logic elements described above are based on interaction between one-by-one propagating pulses, a certain time interval should separate sequential realizations of logic operations. This period provides independent interaction of pulses with nonlinear medium and characterizes a bit rate of the logic gates proposed. In general, it is determined by the relaxation times of the resonant medium. Then the bit rate can be calculated as $1 / T_{2}$, i.e. about $10 \mathrm{Gbit} / \mathrm{s}$. It is not high level, as compared with Kerr-type structures (see, for example, [14]). Nevertheless, in some cases this rate can be increased. Fig. 6a shows propagation of two pulses with $\Omega_{0}=1$ used in OR gate at $\delta=0$. It is seen that time interval of about $\Delta t=200 t_{p}$ between input signals is enough for independent propagation of pulses and operation of OR gate. For the parameters used in simulations we have $t_{p}=30 \mathrm{fs}$ and $\Delta t=6 \mathrm{ps}$, which is much less than relaxation times of the resonant medium. Hence, the rate of logic gates operation, which can be estimated as $1 / \Delta t$, amounts to a value of more than $160 \mathrm{Gbit} / \mathrm{s}$. Obviously, even greater bit rates can be achieved for OR gate due to weak interactions between pulses in this case.

However, this is not the case for AND gate at $\delta=0$. Propagation of two single pulses with $\Omega_{0}=1.5$ separated by the time interval of $\Delta t=200 t_{p}$ does not give two independent low states of AND gate (Fig. 6 b , solid line). This means that pulses interact due to slow relaxation of the medium. As it is seen from Fig. 17a, presence of frequency detuning results in faster relaxation of the dense resonant medium. This can be used to increase bit rate of AND gate. Indeed, the interaction between pulses is significantly weaker for $\delta=1$ (dashed line in Fig. 6b) or even almost absent for $\delta=-1$ (dotted line). Thus, frequency detunings $|\delta| \sim 1$ are useful to realize ultrafast (more than $160 \mathrm{Gbit} / \mathrm{s}$ ) logic operation (in particular, AND). 

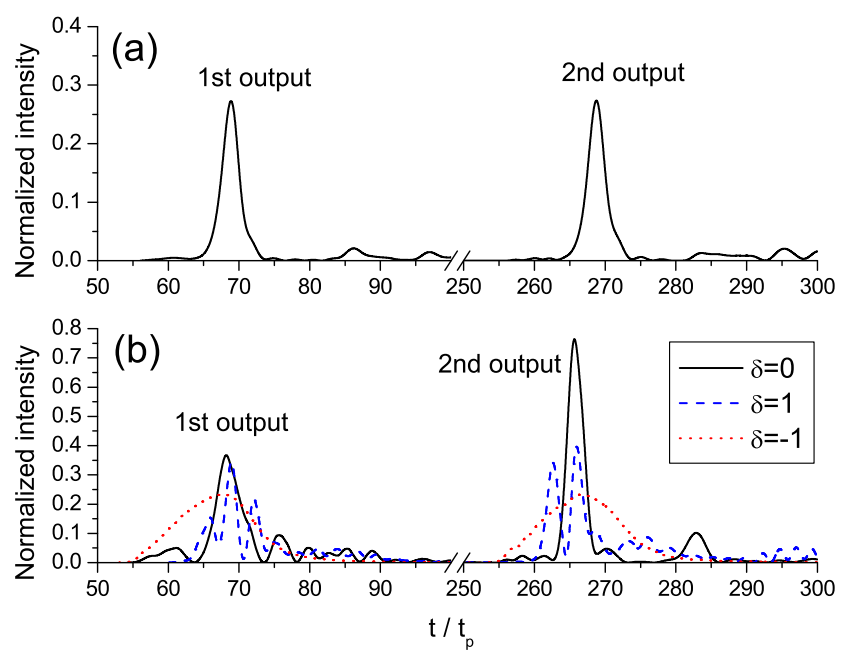

FIG. 6: (Color online) Shapes of transmitted pulses for two sequential single-pulse inputs with time interval of $200 t_{p}$ between them. Input pulse intensities are (a) $\Omega_{0}=1$ at $\delta=0$ (OR gate), (b) $\Omega_{0}=1.5$ at different $\delta$ (AND gate). Other parameters are the same as in Fig. 5

\section{CONCLUSION}

Summing up, propagation effects for light pulses with frequency detuned from the atomic resonance in the dense two-level medium significantly differs from those for resonant radiation. Such important phenomenon as pulse compression shifts towards larger distances, while compensation of dispersion spreading in photonic crystal becomes less pronounced.

Special attention is attracted to possibitity of logic gates realization in nonlinear photonic crystals. The type of logic element is governed by the intensity of input pulses. It turned out that stable and fast OR and NOT gates can be obtained in the system with zero frequency detuning. Detuning becomes necessary in the case of AND gate resulting in fast relaxation and increasing bit rate to the level of more than $160 \mathrm{Gbit} / \mathrm{s}$.

Note that the recompense for simplicity and ultrafast operation of the logic gates considered is high level of peak intensity of input pulses required to observe the effects described above. While, for stationary cases of logic elements [15] or optical switching [8], input intensities are needed to be of about units and tens $\mathrm{kW} / \mathrm{cm}^{2}$, in our case peak intensity reaches values of the order of $\mathrm{GW} / \mathrm{cm}^{2}$. This situation seems to be typical for usage of ultrashort light pulses. The reason is that approximately the same energy as in stationary regime should be transferred in much shorter time in pulse regime.

Can the effects described be really observed? In this paper we investigated this possibility in principle, but not a particular device. Nevertheless, the used characteristics of the dense resonant medium (the relaxation times and the constant $b$ ), which is responsible for nonlinear interaction, are typical for usually discussed candidates - dense gases and excitonic media [3, 7, 12]. At the same time, the parameters of photonic crystal (background refractive indices, layer thicknesses, number of periods) are not so important, because any changes in reflectivity spectrum of the structure can be compensated, for example, by adjusting the thickness of the layers. Thus, though the structure considered is quite hypothetical, there is no reason to say that it is not realistic.
[1] F.A. Hopf, C.M. Bowden, and W.H. Louisell, "Mirrorless optical bistability with the use of the local-field correction", Phys. Rev. A 29, 2591-2596 (1984).

[2] V. Malyshev and E.C. Jarque, "Spatial effects in nonlinear resonant reflection from the boundary of a dense semi-infinite two-level medium: normal incidence", J. Opt. Soc. Am. B 14, 1167-1178 (1997).

[3] A.A. Afanas'ev, R.A. Vlasov, N.B. Gubar, and V.M. Volkov, "Hysteresis behavior in light reflection from a dense resonant medium with intrinsic optical bistability", J. Opt. Soc. Am. B 15, 1160-1167 (1998).

[4] M.E. Crenshaw, M. Scalora, and C.M. Bowden, "Ultrafast intrinsic optical switching in a dense medium of twolevel atoms", Phys. Rev. Lett. 68, 911-914 (1992).

[5] M. Scalora and C.M. Bowden, "Propagation effects and ultrafast optical switching in dense media", Phys. Rev. A 51, 4048-4056 (1995).

[6] C.M. Bowden, A. Postan, and R. Inguva, "Invariant pulse propagation and self-phase modulation in dense media", J. Opt. Soc. Am. B 8, 1081-1084 (1991).

[7] A.A. Afanas'ev, R.A. Vlasov, O.K. Khasanov, T.V. Smirnova, and O.M. Fedorova, "Coherent and incoherent solitons of self-induced transparency in dense, resonant media", J. Opt. Soc. Am. B 19, 911-919 (2002).

[8] D.V. Novitsky and S.Yu. Mikhnevich, "Bistable behavior of reflection and transmission of a one-dimensional photonic crystal with a dense resonant medium as a defect", J. Opt. Soc. Am. B 25, 1362-1370 (2008).

[9] R. Friedberg, S.R. Hartmann, and J.T. Manassah, "Effect of local-field correction on a strongly pumped resonance", Phys. Rev. A 40, 2446-2451 (1989).

[10] M.E. Crenshaw, "Quasiadiabatic approximation for a dense collection of two-level atoms", Phys. Rev. A 54, 3559-3575 (1996).

[11] C. Bowden and J.P. Dowling, "Near dipole-dipole effects in dense media: Generalized Maxwell-Bloch equations", Phys. Rev. A 47, 1247-1251 (1993).

[12] D.V. Novitsky, "Compression of an intensive light pulse in photonic-band-gap structures with a dense resonant medium", Phys. Rev. A 79, 023828 (2009). ArXiv: 0908.3777.

[13] K. Asakawa et al., "Photonic crystal and quantum dot technologies for all-optical switch and logic device", New J. Phys. 8, 208 (2006). 
[14] P. Andalib and N. Granpayeh, "All-optical ultracompact photonic crystal AND gate based on nonlinear ring resonators", J. Opt. Soc. Am. B 26, 10-16 (2009).

[15] I. Nefedov, Y. Morozov, V. Gusyatnikov, and A.
Zheltikov, "Optically controlling photonic band gap logic element", 2nd International Conference on Transparent Optical Networks, 195-198 (2000). 